\title{
Clinical characteristics and treatment of different types of cesarean scar pregnancy
}

\author{
Xuai Yin ${ }^{1}\left[\right.$, Shihai Huang ${ }^{2}$ (]) \\ ${ }^{1}$ Affiliated Chaohu Hospital of Anhui Medical University, Hefei, Anhui, Department of Obstetrics and Gynecology, PR China \\ ${ }^{2}$ Affiliated Chaohu Hospital of Anhui Medical University, Hefei, Anhui, Department of Ultrasonography, PR China
}

\begin{abstract}
Objectives: The purpose of this study was to evaluate the clinical characteristics and compare the treatment efficacy of different types of cesarean scar pregnancy (CSP).

Material and methods: We performed a retrospective chart review of 66 women (69 cases) with CSP who received treatment with mifepristone/methotrexate (MTX) plus curettage, uterine artery embolization (UAE) plus curettage, additional MTX, or laparotomy, and compared the clinical characteristics, treatment efficacy, and occurrence of complications among 3 types of CSP (partial, complete, and mass type).

Results: Review of the 69 cases revealed a considerable increase of gestational duration $(p<0.001)$, sac/lesion size $(p<0.001)$ and vaginal bleeding $(p<0.05)$ in patients with mass-type CSP compared to that of other types. All CSP cases were successfully treated, 4 cases of mass-type received laparotomy and none of the cases required a hysterectomy. Severe bleeding was observed in 2 cases of partial-type and complete-type, respectively, and 3 cases for mass-type. Moreover, bleeding occurred during initial treatment with mifepristone plus curettage in partial-type cases, but not with UAE plus curettage.

Conclusions: UAE plus curettage is a more effective treatment option for partial-and complete-type of CSP than mifepristone plus curettage. The cases of mass-type often need surgery and are prone to have longer gestational duration, larger lesions, and more vaginal bleeding.
\end{abstract}

Key words: cesarean scar pregnancy; clinical characteristics; treatment efficacy; complication

Ginekologia Polska 2020; 91, 7: 406-411

\section{INTRODUCTION}

The increasing rate of cesarean section has attracted worldwide concerns in recent decades [1]. The post-cesarean era and requirement of two-child policy in China has made the situation worse, although the occurrence of cesarean section without medical indication was controlled. Long-term complications of a cesarean section, such as diverticulum which induced prolonged menstruation and irregular vaginal bleeding, the complications and treatment used in partial, complete, and mass-type CSP cases were shown in Table 1. The most severe complication, cesarean scar ectopic pregnancy (CSP) which was defined as an ectopic pregnancy in which the fertilized egg is implanted in the previous cesarean section scar, may cause uncontrollable bleeding, uterine rupture, hysterectomy, placenta previa, and can be life-threatening [2]. CSP was reported to be sporadic in case reports decades ago [3, 4]; however, in recent years there have been increased reports of CSP emerged, including randomized control trials (RCTs) [5-10], cohort studies [11-13], and comparative case studies [14]. Thus, CSP is no longer a rare form of ectopic pregnancy.

\begin{tabular}{|l|l|}
\hline \multicolumn{2}{|l|}{ Table 1. Clinical features of the 69 CSP cases $(\mathrm{n}=66)$} \\
\hline Characteristic & Mean \pm SD (\%) \\
\hline Age $[\mathrm{y}]$ & $32.39 \pm 4.25$ \\
\hline No. of curettage $[\mathrm{n}]$ & $1.75 \pm 1.75$ \\
\hline No. of prior cesarean delivery [n] & $1.41 \pm 0.52$ \\
\hline Time since previous cesarean delivery, $\mathrm{y}[\%]$ & $1(10.14 \%) \geq 2(89.86 \%)$ \\
\hline $\begin{array}{l}\text { Gestational weeks at time of diagnosis } \\
\text { [weeks] }\end{array}$ & $7.75 \pm 2.14(5-16)$ \\
\hline Presence of fetal heartbeat [n] & $29.16 \pm 17.43(5-74)$ \\
\hline Abdominal pain [n] & $7(10.14 \%)$ \\
\hline Vaginal bleeding & $39(56.5 \%)$ \\
\hline Data are presented as $n$, mean \pm SD, median (range), or $n(\%)$
\end{tabular}




\begin{tabular}{|c|c|c|c|c|}
\hline Characteristic & Partial & Complete & Mass & p-value \\
\hline Case number [n] & 33 & 28 & 8 & \\
\hline $\begin{array}{l}\text { Age [y] } \\
(\text { mean } \pm S D)^{*}\end{array}$ & $32.1 \pm 4.4$ & $33.2 \pm 4.2$ & $30.9 \pm 3.6$ & 0.326 \\
\hline $\begin{array}{l}\text { No. of curettage } \\
(\text { mean } \pm S D)^{*}\end{array}$ & $1.30 \pm 1.29$ & $1.25 \pm 1.17$ & $1.50 \pm 1.41$ & 0.884 \\
\hline $\begin{array}{l}\text { No. of prior } \\
\text { cesarean delivery } \\
(\text { mean } \pm S D)^{*}\end{array}$ & $1.36 \pm 0.55$ & $1.50 \pm 0.51$ & $1.25 \pm 0.46$ & 0.407 \\
\hline \multicolumn{5}{|l|}{$\begin{array}{l}\text { Time since } \\
\text { previous } \\
\text { cesarean delivery } \\
\text { [y] }\end{array}$} \\
\hline 1 & 4 & 3 & 0 & 0.867 \\
\hline$\geq 2$ & 29 & 25 & 8 & \\
\hline $\begin{array}{l}\text { Gestational weeks } \\
(\text { mean } \pm S D)^{*}\end{array}$ & $7.24 \pm 1.58$ & $7.43 \pm 1.75$ & $11.0 \pm 2.78$ & $<0.001^{*}$ \\
\hline $\begin{array}{l}\text { Presence of fetal } \\
\text { heartbeat }{ }^{\#}\end{array}$ & 13 & 7 & 0 & 0.273 \\
\hline Abdominal pain" & 2 & 4 & 1 & 0.442 \\
\hline Vaginal bleeding ${ }^{\#}$ & 18 & 13 & 8 & $0.019^{*}$ \\
\hline
\end{tabular}

Currently, to the best of our knowledge, the consensus or guidelines on the diagnosis and treatment of CSP is unclear. Hence, a retrospectively study involving 69 cases of CSP (partial-, complete-, and mass-type CSP) was conducted to compare the clinical characteristics, treatment efficacy, and occurrence of complications among the different types.

\section{MATERIAL AND METHODS}

This is a retrospective case series of patients with diagnosis of CSP who treated at Chaohu Hospital (the Affiliated Chaohu Hospital of Anhui Medical University, Hefei, Anhui, PR China) from January 2016 to January 2019. Thirteen women were excluded because the absence of related clinical characteristics, lost for follow-up or unclear ultrasound images, and 79 women diagnosed with CSP were selected based on the following criteria: clear ultrasound images, no absence of data or follow-up. Sixty-six women with CSP (69 cases) met the criteria were enrolled in the study. This study was approved by the Ethics Committee of the hospital (approval no. 201901-kyxm-02).

Clinical characteristics of the patients are summarized in Table 2 which include age, gestational weeks, pregnancy duration, abortion and curettage time, cesarean section time, interval since previous cesarean section, gestational sac/lesion size, fetal heartbeat, initial serum $\beta$-human chorionic gonadotropin $\beta$-(hCG) level, vaginal bleeding, and abdominal pain. The ultrasonographic images were evaluated based on the criteria [15], the abdominal and vaginal ultrasonographic instruments used were Mylab 90 of Baisheng (made in China) and GE-LOGIQ E9(made in America). According to the classification rules [16], diagnosis and treatment consensus [17] and mass type of CSP commonly observed in clinic [18], the 69 cases were divided into 3 types: partial-, complete-, and mass-type. The partial-type is defined when the gestational sac is partially embedded in the myometrium of the scar, and a part or most of the gestational sac has grown toward the endometrial cavity, with the gestational sac deformed, elongated, with a sharp angle at the lower end, and surrounded by vasculature. In complete-type CSP, the gestational sac is totally embedded in the myometrium of the scar, with an empty endometrial cavity and cervical canal surrounded by vasculature. Mass-type CSP is defined when there are mixed echoes located in the scar of the lower uterine segment, protruding toward the bladder, with the local muscular layer thinned, and abundant blood flow surrounding it.

The clinical characteristics, treatment efficacy, and occurrence of complications were compared in all cases. Successful treatment was considered as a reduction or disappearance of the mass and/or significant normalization of the serum $\beta$-hCG level.

Several therapeutic approaches used for these cases involving mifepristone or methotrexate (MTX) plus curettage; uterine artery embolization (UAE) plus curettage; additional MTX; and laparotomy treatment. Curettage was performed via the method of dilatation and suction with the guidance of ultrasound. The embolic agents used in UAE were gelatin sponge granules and sponge strips

Statistical analysis was performed using SPSS version 19.0. Continuous variables were expressed as means standard deviations for normal distribution and median for non-normal distribution, statistical significance was tested using Student's t-test and Mann-Whitney U-test, respectively. One-way ANOVA followed by Bonferonni's test was used to compare the difference among groups. Categorical data were presented as frequencies or percentages and compared by Chi-square test or Fisher's exact test. A two-sided $p$-value of 0.05 was considered statistical significance.

\section{RESULTS}

From January 2016 to January 2019, 69 cases of CSP(66 patients) were managed in our unit (1 case of partial-type and 2 cases of complete-type turned into mass-type CSP after treatment, the initial and subsequent treatment of these 3 cases were conducted in Chaohu Hospital). The clinical characteristics of 69 cases are shown in Table 2 . Of the 69 cases, 1 of them with partial-type CSP became pregnant 11 months after treatment with mifepristone-progesterone and curettage, additional methotrexate (MTX) with second curettage; she delivered a healthy female infant weighing $3675 \mathrm{~g}$ with an Ap- 

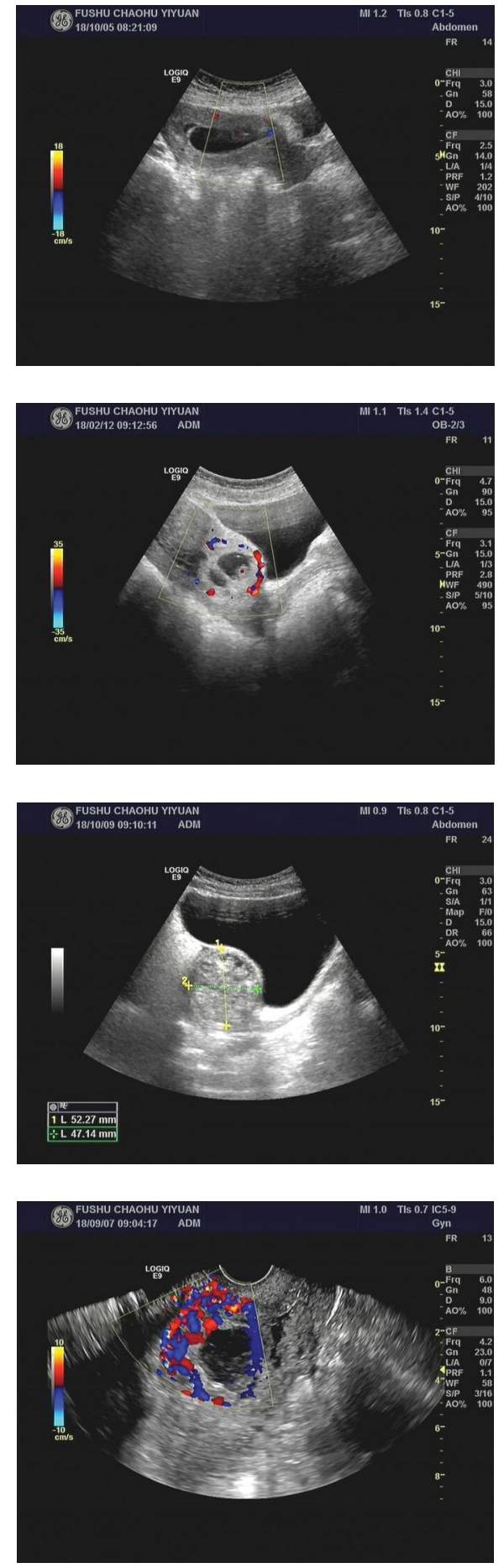

Figure 1. Ultrasound images of the 3 types of CSP. A, partial type. B, complete type. $C$ and $D$, mass type

gar score of 9 at $1 \mathrm{~min}$ and 10 at 5 min via cesarean section at term. Placenta previa and partial adhesion were observed during the operation, and the estimation of blood loss was $400 \mathrm{~mL}$. Another case was a recurrent scar pregnancy diagnosed in 15 months ago, where she underwent treatment with mifepristone combined with uterine curettage.
Sixty-nine cases of CSP, including 33 partial-type cases, 28 complete-type cases, and 8 mass-type. Among the 8 mass-type cases, 1 case was diagnosed by transvaginal ultrasound, presenting menopause of 50 days with vaginal bleeding. The other 7 cases were diagnosed after medical intervention such as suction curettage or drug-induced abortion, and the time of diagnosis varies from hours to 2 months. Out of 7 cases 3 received initial and subsequent treatment at the same hospital, whereas the other 4 cases received initial treatment at other hospitals. The ultrasonographic images of the 3 types of CSP were shown in Figure 1.

The clinical characteristics of patients among the 3 CSP subgroups were shown in Table 2 . We found there were no significant differences in age, curettage times, cesarean section times and intervals, gestational weeks, cardiac activities, gestational sac/lesion size, vaginal bleeding, and abdominal pain between partial- and complete-type CSPs. Compare to the partial- and complete-type of CSP, the gestational duration was significantly increased $(p<0.001)$, so was the sac/lesion size $(p<0.001)$ and vaginal bleeding $(p<0.01)$ in mass-type patients, but there was no significant difference in age, curettage times, cesarean section times and intervals, as well as abdominal pain.

All of 69 CSP cases were successfully treated with one or more combined treatments. There were 12 of 33 cases with partial-type CSP initially treated with mifepristone plus curettage, and 10 cases were successful treated in which 2 cases received additional MTX, moreover, 2 cases received UAE due to bleeding during the treatment. The success rate of treatment with mifepristone plus uterine curettage \pm MTX in partial-type CSP was $83.3 \%$ (10/12). The initial treatment with UAE plus curettage of twenty cases were successful which presented no excessive bleeding. Therefore, the success rate of UAE plus curettage in partial-type CSP was $100.0 \%$ (20/20). One case referred from other institutions had been previously misdiagnosed as intrauterine pregnancy and artificial abortion was performed directly. After that, the repeated vaginal bleeding occurred for 2 months until the second time to hospital and was misdiagnosed with trophoblastic neoplasm before the surgical treatment was performed, it has been confirmed as CSP by the intraoperative findings and postoperative pathology.

In the group with complete-type of CSP, 6 of 28 first-consulted patients were successfully treated with drug plus curettage ( 4 with mifepristone, and 2 with MTX). The other 22 were treated with UAE and curettage initially in which 20 cases (including 1 case that accepted additional MTX) underwent successful treatment, whereas 2 had a stable pregnancy (mass-type) later. As a result, the success rate of UAE plus curettage with or without MTX in complete-type CSP was $90.9 \%(20 / 22)$. 
In the group with mass-type of CSP, 4 of 8 were treated with UAE plus curettage and 1 with additional MTX. The laparotomy was performed in other 4 cases; 1 case was the preceding partial-type CSP which was misdiagnosed initially, and turned into a mass-type after a medical abortion, and the other 3 cases treated with MTX, of which 2 were preceding complete-type CSP which underwent treatment of UAE plus uterine curettage. All 4 surgeries were performed by laparotomy and scar repair, and no severe bleeding was observed during operation.

As one of the major complications, hysterectomy had not been observed in any cases of our series, the complications and treatment used in partial, complete, and mass-type CSP cases were shown in Table3. As showed in our data, moderate bleeding (> $200 \mathrm{~mL}$ ) occurred in 4 partial-type, 3 complete-type, and 1 mass-type case, while severe bleeding ( $>500 \mathrm{~mL}$ ) occurred in 2 partial-type, 2 complete-type, and 3 mass-type cases. However, patients of partial-type experienced bleeding during the initial treatment of mifepristone combined with curettage, but not for the treatment regimen of UAE plus curettage which showed no severe bleeding. In 2 complete-type and 3 mass-type of patients developed bleeding during the UAE plus curettage \pm MTX.

\section{DISCUSSION}

In our study, we found that vaginal bleeding is the most common symptom among patients in CSP. Mass-type CSP cases are prone to have longer gestational duration, larger lesions, and more vaginal bleeding than partial- and complete-type. In addition, we noted that UAE plus curettage is a more effective therapeutic approach than mifepristone plus curettage which is the basic treatment for CSP. Moreover, most of the mass-type CSPs were developed from complete- and partial-type CSP after medical treatment and surgery is unavoidable to remove lesions.
A retrospective case-control study by Luo et al. [19] has shown that $58.7 \%$ of CSP cases experience vaginal bleeding and $15 \%$ experience abdominal pain as early symptoms. Kim et al. [20] reported that $53.4 \%$ of CSP cases had no symptoms around gestational weeks $6.5 \pm 1.1$. In our study, 39 (56.5\%) cases experienced vaginal bleeding and 7 cases (10.14\%) experienced abdominal pain, which were consistent with the previous reports. Approximately half of the CSP patients had no obvious early symptoms, based on these reports and our results we strongly recommended to pay more attention to patients after cesarean section, especially when they experience vaginal bleeding. Timor-Tritsch et al. [21] have suggested that routine ultrasound screening for CSP should be performed in pregnant women with a history of cesarean section at gestational weeks 5-7 like screening for aneuploidy and preeclampsia. Early detection of CSP is critical to prevent its progression and improve the long-term prognoses.

The clinical features of CSP differ among different types. Our study showed that there were no significant differences in age, curettage times, cesarean section times and intervals, gestational weeks, cardiac activities, gestational sac/lesion size, vaginal bleeding, and abdominal pain between partial-and complete-type CSP. However, the results indicated that the characteristics of mass-type CSP involving the occurrence in later gestational week, a larger lesion, vaginal bleeding tendency, and it often develops from partial- and complete-type CSP after medical treatment, which is consistent with the previous report of Ying et al. [22].

To the best of our knowledge, CSP represents a diagnostic and therapeutic challenge for the unclear guidelines of the classification and treatment till date. Our study showed that in partial-type CSP, the success rate of treatment with mifepristone plus curettage \pm MTX was $83.3 \%$ (10/12), and 17 cases were successfully treated with UAE plus curettage. The success rate of treatment with UAE plus curettage \pm MTX in complete-type CSP was $90.9 \%$ (20/22). This result was

\begin{tabular}{|c|c|c|c|c|c|c|c|}
\hline \multirow{2}{*}{ Type } & \multirow{2}{*}{ Initial treatment (n) } & \multirow{2}{*}{$\begin{array}{l}\text { Successful } \\
\text { with no } \\
\text { intervention }\end{array}$} & \multirow{2}{*}{$\begin{array}{l}\text { Successful with } \\
\text { additional MTX }\end{array}$} & \multirow{2}{*}{$\begin{array}{l}\text { Success rate } \\
\text { (initial treatment } \\
\text { + additional MTX) }\end{array}$} & \multirow{2}{*}{$\begin{array}{l}\text { Eventual success with } \\
\text { UAE or laparotomy, } \\
\text { or became mass type }\end{array}$} & \multicolumn{2}{|c|}{ Bleeding ( $\mathrm{mL}$ ) } \\
\hline & & & & & & \multicolumn{2}{|c|}{$>200>500$} \\
\hline \multirow{3}{*}{ Partial } & Mifepristone plus curettage (12) & 7 & 3 & $83.3 \%(10 / 12)$ & $\operatorname{UAE}(2)$ & 4 & 2 \\
\hline & UAE plus curettage (0) & 20 & 0 & $100 \%(20 / 20)$ & 0 & 0 & 0 \\
\hline & Direct induced abortion (1) & 0 & & & Turned into mass ( 1 ) & & \\
\hline \multirow{3}{*}{ Complete } & Mifepristone plus curettage (4) & 4 & & & 0 & 2 & 0 \\
\hline & Additional MTX plus curettage (2) & 2 & & & 0 & 1 & 0 \\
\hline & UAE plus curettage (22) & 19 & 1 & $90.9 \%(20 / 22)$ & Turned into mass (2) & 0 & 2 \\
\hline \multirow{3}{*}{ Mass } & UAE plus curettage (4) & 3 & 1 & 1 & & 1 & 1 \\
\hline & MTX (3) & 0 & & & laparotomy (3) & 0 & 2 \\
\hline & Laparotomy(1) & 1 & & & & 0 & 0 \\
\hline
\end{tabular}


consistent with that of previous reports [7, 23]. However, the safety of the patients, who underwent UAE treatment, to become pregnant again is under controversial. The risks include ovarian failure, infertility, abortion, premature delivery, and postpartum hemorrhage [24, 25]. However, in other cases, such as partial-type CSP with a thicker musculature, UAE should be used when emergency massive hemorrhage occurs or as a second-line treatment instead of routine treatment.

The curative effect didn't show an increase with the treatment of uterine curettage followed by MTX, in addition, it showed the ability to prolong the length of hospitalization [26]. MTX should not be used as an initial treatment because the fibrous tissue surrounding the gestational sac reduces the absorption and effectiveness of MTX [27]. However, as a remedy, MTX has a certain success rate due to the viability of trophoblasts. In our study, 4 of 6 cases of partial- and complete-type CSP received additional MTX which had been successfully treated.

A previous report [12] has shown that the thinner the uterine myometrium between the gestational sac and the bladder, the treatment would be more difficult, and the more likely to have a surgery with more complications. Because there was no description of muscle thickness in some images in our retrospective study, it was not possible to further classify complete-type CSP.The mass-type in our study appeared to be a large lesion, protruding toward the bladder, with a thin muscular wall and abundant blood flow. In our study, 4 of 8 cases with mass-type CSP underwent UAE; 3 were successfully treated and 1 required additional MTX with success. The remaining 4 were eventually treated surgically. In our series, three cases of mass-type CSP developed severe bleeding during treatment with UAE plus curettage and MTX, but not with an operation. Therefore, for the treatment of mass-type CSP, surgery was the most recommended option to remove the lesions because it would increase the possibility to bleeding and prolong the recovery time.

The mean gestational duration in our study was $7.75 \pm 2.14$ weeks, and an early diagnosis contributed to the early treatment. None of the 69 cases underwent hysterectomy, and seven of 8 cases of mass-type CSP developed after abortion or drug interference. Therefore, $\beta$-hCG levels and ultrasound should be monitored regularly after abortion of scar pregnancy in case the occurrence of pregnancy. Surgery is unavoidable once mass-type CSP develops. Although surgical treatment is considered the optimal treatment choice, medical drug plus curettage can achieve similar results for patients who have non-mass-type CSP with a thicker scar and with low economic status to undergo surgery. Thus, how to remove the pregnancy safety and effectively, and to reduce the bleeding and injury to prevent hysterectomy in CSP cases are need for further study.
There are some limitations in our study. There is no description of muscle thickness and blood flow in some images in our retrospective study, it is not possible to further typing complete CSP. We did not make comparisons of $\beta$-hCG for the levels of $\beta$-hCG (e.g. $>10,000 \mathrm{IU} / \mathrm{L}$, other than a defined figure such as $12,000 \mathrm{mIU} / \mathrm{mL}$ ) were not given for some CSP patients. In addition, the therapeutic approaches of UAE in partial-type CSP were slightly more, and which may affect credibility to some extent.

In conclusion, UAE combined with curettage is a more effective treatment instead of mifepristone plus curettage, which is the basic treatment for partial- and complete-type CSP. Mass-type CSP has features such as occurrence at late gestational duration, larger lesion size, and more vaginal bleeding tendency. Most mass-type CSPs develop from partial- and complete-type CSPs after medical treatment, and many cases require surgery to remove the lesions. Improper treatment may induce the increase of bleeding and impede the patients' recovery.

\section{Author contributions}

Design, planning, methodology, conduct, validation, data analysis and manuscript writing: Xuai Yin; Validation: Shihai Huang.

\section{Acknowledgments}

We thank all the staff of Department of Obstetrics and Gynecology and archives at the Affiliated Chaohu Hospital of Anhui Medical University, Hefei, Anhui, PR China.

\section{Conflicts of interest}

The authors have no conflicts of interest.

\section{Ethical Statement}

This manuscript has not been published or presented elsewhere in part or in entirety and is not under consideration by another journal. All study participants provided informed consent, and the study design was approved by the appropriate ethics review board. We have read and understood your journal's policies, and we believe that neither the manuscript nor the study violates any of these.

\section{REFERENCES}

1. Souza JP, Gülmezoglu Am, Lumbiganon P, et al. WHO Global Survey on Maternal and Perinatal Health Research Group. Caesarean section without medical indications is associated with an increased risk of adverse short-term maternal outcomes: the 2004-2008 WHO Global Survey on Maternal and Perinatal Health. BMC Med. 2010; 8: 71, doi: 10.1186/17417015-8-71, indexed in Pubmed: 21067593.

2. Calì G, Timor-Tritsch IE, Palacios-Jaraquemada J, et al. Outcome of Cesarean scar pregnancy managed expectantly: systematic review and meta-analysis. Ultrasound Obstet Gynecol. 2018; 51(2): 169-175, doi: 10.1002/uog.17568, indexed in Pubmed: 28661021.

3. Larsen JV, Solomon MH. Pregnancy in a uterine scar sacculus--an unusual cause of postabortal haemorrhage. A case report. S Afr Med J. 1978; 53: $142-143$. 
4. Smith A, Ash A, Maxwell D, et al. Caesarean scar pregnancy. BJOG. 2007; 114(3): 253-263, doi: 10.1111/j.1471-0528.2006.01237.x, indexed in Pubmed: 17313383

5. Peng P, Gui T, Liu X, et al. Comparative efficacy and safety of local and systemic methotrexate injection in cesarean scar pregnancy. Ther Clin Risk Manag. 2015; 11: 137-142, doi: 10.2147/TCRM.S76050, indexed in Pubmed: 25670903.

6. Qian ZD, Weng Y, Du YJ, et al. Risk factors for intraoperative hemorrhage at evacuation of a cesarean scar pregnancy following uterine artery embolization. Int J Gynaecol Obstet. 2013; 123(3): 240-243, doi: 10.1016/j. ijgo.2013.06.029, indexed in Pubmed: 24054055.

7. Zhuang $Y$, Huang L. Uterine artery embolization compared with methotrexate for the management of pregnancy implanted within a cesarean scar. Am J Obstet Gynecol. 2009; 201(2): 152.e1-152.e3, doi: 10.1016/j.ajog.2009.04.038, indexed in Pubmed: 19527897.

8. Li C, Li C, Feng D, et al. Transcatheter arterial chemoembolization versus systemic methotrexate for the management of cesarean scar pregnancy. Int J Gynaecol Obstet. 2011;113(3): 178-182, doi: 10.1016/j. ijgo.2010.11.027, indexed in Pubmed: 21458810.

9. Wang M, Yang Z, Li Y, et al. Conservative management of cesarean scar pregnancies: a prospective randomized controlled trial at a single center. Int J Clin Exp Med. 2015; 8(10): 18972-18980, indexed in Pubmed: 26770522.

10. Li Y, Gong L, Wu X, et al. Randomized controlled trial of hysteroscopy or ultrasonography versus no guidance during $D \& C$ after uterine artery chemoembolization for cesarean scar pregnancy. Int J Gynaecol Obstet. 2016; 135(2): 158-162, doi: 10.1016/j.ijgo.2016.04.019, indexed in Pubmed: 27634054.

11. Harb HM, Knight $M$, Bottomley $C$, et al. Caesarean scar pregnancy in the UK: a national cohort study. BJOG. 2018; 125(13): 1663-1670, doi: 10.1111/1471-0528.15255, indexed in Pubmed: 29697890.

12. Lin SY, Hsieh CJ, Tu YA, et al. New ultrasound grading system for cesarean scar pregnancy and its implications for management strategies: An observational cohort study. PLoS One. 2018; 13(8): e0202020, doi: 10.1371/journal.pone.0202020, indexed in Pubmed: 30092014.

13. He Y, Wu X, Zhu Q, et al. Combined laparoscopy and hysteroscopy vs. uterine curettage in the uterine artery embolization-based management of cesarean scar pregnancy: a retrospective cohort study. BMC Womens Health. 2014; 14: 116, doi: 10.1186/1472-6874-14-116, indexed in Pubmed: 25248928.

14. Birch Petersen K, Hoffmann E, Rifbjerg Larsen C, et al. Cesarean scar pregnancy: a systematic review of treatment studies. Fertil Steril. 2016; 105(4): 958-967, doi: 10.1016/j.fertnstert.2015.12.130, indexed in Pubmed: 26794422

15. Fylstra D. Ectopic Pregnancy Within a Cesarean Scar: A Review. Obstetrical \& Gynecological Survey. 2002; 57(8): 537-543, doi: 10.1097/00006254-200208000-00024.
16. Vial Y, Petignat $P$, Hohlfeld P. Pregnancy in a cesarean scar. Ultrasound Obstet Gynecol. 2000; 16(6): 592-593, doi: 10.1046/j.1469-0705.2000.0 0300-2.x, indexed in Pubmed: 11169360.

17. Family planning group of obstetrics and gynecology branch, Chinese medical association. Expert consensus on diagnosis and treatment of cesarean scar pregnancy. Chinese J Obstet Gynecol. 2016; 51:568-571.

18. Liu Z, Dai Q, Wang M, et al. Clinical and ultrasonic characteristics of cesarean scar pregnancy shown as lower uterine segment mass. Chinese J Med Imag. 2013; 29: 1006-1010.

19. Luo L, Ruan X, Li C, et al. Early clinical features and risk factors for cesarean scar pregnancy: a retrospective case-control study. Gynecol Endocrinol. 2018; 35(4):337-341, doi: 10.1080/09513590.2018.1526276.

20. Kim SoY, Yoon SoRa, Kim MiJ, et al. Cesarean scar pregnancy; Diagnosis and management between 2003 and 2015 in a single center. Taiwan J Obstet Gynecol. 2018; 57(5): 688-691, doi: 10.1016/j. tjog.2018.08.013, indexed in Pubmed: 30342652.

21. Timor-Tritsch IE, D'Antonio F, Calí G, et al. Early first-trimester transvaginal ultrasound is indicated in pregnancy after previous Cesarean delivery: should it be mandatory? Ultrasound Obstet Gynecol. 2019; 54(2): 156-163, doi: 10.1002/uog.20225, indexed in Pubmed: 30677186.

22. Ying $X$, Zheng $W$, Zhao $\mathrm{Li}$, et al. Clinical characteristics and salvage management of persistent cesarean scar pregnancy. J Obstet Gynaecol Res. 2017; 43(8): 1293-1298, doi: 10.1111/jog.13367, indexed in Pubmed: 28612958

23. Tumenjargal A, Tokue H, Kishi H, et al. Uterine Artery Embolization Combined with Dilation and Curettage for the Treatment of Cesarean Scar Pregnancy: Efficacy and Future Fertility. Cardiovasc Intervent Radiol. 2018; 41(8): 1165-1173, doi: 10.1007/s00270-018-1934-z, indexed in Pubmed: 29546456

24. Walker WJ, McDowell SJ. Pregnancy after uterine artery embolization for leiomyomata: a series of 56 completed pregnancies. Am J Obstet Gynecol. 2006; 195(5): 1266-1271, doi: 10.1016/j.ajog.2006.04.011, indexed in Pubmed: 16796984.

25. Mohr-Sasson A, Spira M, Rahav R, et al. Ovarian reserve after uterine artery embolization in women with morbidly adherent placenta: A cohort study. PLoS One. 2018; 13(11): e0208139, doi: 10.1371/journal. pone.0208139, indexed in Pubmed: 30496259.

26. Wang S, Beejadhursing $\mathrm{R}, \mathrm{Ma} \mathrm{X}$, et al. Management of Caesarean scar pregnancy with or without methotrexate before curettage: human chorionic gonadotropin trends and patient outcomes. BMC Pregnancy Childbirth. 2018; 18(1): 289, doi: 10.1186/s12884-018-1923-x, indexed in Pubmed: 29973177.

27. Liu W, Shen L, Wang $Q$, et al. Uterine artery embolization combined with curettage vs. methotrexate plus curettage for cesarean scar pregnancy. Arch Gynecol Obstet. 2016; 294(1): 71-76, doi: 10.1007/s00404-0153952-2, indexed in Pubmed: 26581398. 